\title{
Psychophysical features of the transition from pure heat perception to heat pain perception
}

\author{
STEFAN LAUTENBACHER, ANDREAS MÖLTNER, and FRIEDRICH STRIAN \\ Max Planck Institute of Psychiatry, Clinical Institute, Munich, Germany
}

\begin{abstract}
The psychophysical features of the transition from the pure heat to the heat pain range were studied in 25 healthy subjects (mean age 28.8 years). Thirty short heat stimuli from $-1.6^{\circ} \mathrm{C}$ to $+1.6^{\circ} \mathrm{C}$ relative to the pain threshold were applied to the thenar of the left hand with an apparatus containing a Peltier thermode (nine different temperatures at $0.4^{\circ} \mathrm{C}$ intervals). The subjects rated the sensation intensity on a visual analogue scale. The resulting stimulus/sensation intensity relations could be explained equally well (same goodness of fit) by a model with a power function (PF) and by a model with two linear regression lines (TLR), one for stimulus intensities below and one for those above the pain threshold and intersecting at the pain threshold. The slopes of the TLR model were significantly larger above the pain threshold than below it. The PF model produced exponents between 1.8 and 1.9. We conclude that to describe the transition area, it is sufficient to use simple linear models for both the pure heat and the heat pain ranges.
\end{abstract}

Since Stevens's (1960) innovative approaches to psychophysics, the relation between stimulus intensity and sensation intensity has traditionally been expressed as a power function. This has been true also for the special case of the psychophysics of pain, where power functions have seemed to provide the best available models. The exponent of the power function has been interpreted as the capacity of the pain system to encode differing stimulus intensities in the pain range. But numerous studies on this topic have demonstrated that the exponent also depends on many other, mainly methodological, conditions, such as the type of power function (with or without threshold corrections; with or without an additional additive constant), the type of data (linear or logarithmic), the type of curve-fitting procedure (simple least squares procedure, or a variation of that procedure), the type and range of stimulus and sensation scaling, and the use of a modulus (Bromm \& Treede, 1980; Cross, Tursky, \& Lodge, 1975; Jones \& Gwynn, 1984; Rollman \& Harris, 1987; Stam, Petrusic, \& Spanos, 1981).

With electrocutaneous stimulation, the most common type used in pain psychophysics, the estimated exponents have varied widely, from 0.7 to 3.5 (Beck \& Rosner, 1968; Stevens, Carton, \& Shickman, 1958), but in the more recent studies they have been concentrated in the range of 1.0 to 1.5 (Algom, Raphaeli, \& Cohen-Raz, 1986, 1987; Bromm \& Treede, 1980; Jones \& Gwynn, 1984; Rollman \& Harris, 1987).

This research was supported by Grant Str 273/3 from the Deutsche Forschungsgemeinschaft. We wish to thank Maria Craig for her valuable comments on eariier versions of this manuscript. Correspondence should be addressed to S. Lautenbacher, Max Planck Institute of Psychiatry, Kraepelinstr. 10, 8000 Munich 40, Germany.
Studies on thermal pain have been less frequent. They have seemed to yield different results, depending on whether radiation or contact heating techniques have been used (Price, 1988). In a series of studies with radiation heating, Adair and coworkers (Adair, Stevens, \& Marks, 1968) found an exponent near unity, whereas in several experiments with contact heating, Price and coworkers (Price, 1988; Price, Barrell, \& Gracely, 1980; Price \& Harkins, 1987; Price, McGrath, Rafii, \& Buckingham, 1983) observed a much more expansive power function with exponents of about 2.1 .

We think that the contact heating experiments yielded an overestimation of the exponent. First, they involved a power function with a threshold correction, but the threshold was the base temperature of the procedure, $34^{\circ} \mathrm{C}$ or $35^{\circ} \mathrm{C}$. And Adair et al. (1968) have shown convincingly that with threshold corrections far below the real pain threshold, the exponent is overestimated, due alone to the formal properties of power functions. In a recent study, an exponent of 2.1 was also found with radiation heating, but again probably only because the clearly nonpainful temperature of $35^{\circ} \mathrm{C}$ was used as the "threshold" (Campbell, Carstens, \& Watkins, 1991). Second, and more relevant to the present study, the psychophysical function spanned the range from $34^{\circ} \mathrm{C}$ or $35^{\circ} \mathrm{C}$ to clearly painful stimulus intensities; that is, it covered the warmth, pure heat, and heat pain ranges, not just the heat pain range. But data presented by LaMotte and Campbell (1978) suggest that the slope of the psychophysical function is close to linear both in the heat range and in the heat pain range and that an increase in slope occurs in the region of the pain threshold. A power function can be fitted to such data only if it can have an exponent that is considerably greater than unity. 
The apparent inconsistencies in the results just summarized led us to examine more closely the transition from pure heat perception to heat pain perception. We suspected that it was mainly the inclusion by Price and coworkers of this transition area in their psychophysical function that produced the exponent around 2.1 and not the use of contact heating. Our assumptions were as follows: (1) The psychophysical function that best describes the stimulusintensity/sensation-intensity relationship in the transition area consists of two regression lines, one for the stimuli below the pain threshold and one for those above it; and (2) the slope of the regression line for the pure heat range is less steep than the slope of the one for the heat pain range. To test these assumptions, we developed a psychophysical model with two linear regression lines intersecting at the pain threshold. We then compared this model as to goodness of fit with one using the traditional power function with a threshold correction for the same stimulus range. Thus, we compared two models with three free parameters each (the number of parameters constitutes another influence on the goodness of fit; see Beck \& Rosner, 1968; McCallum \& Goldberg, 1975). We used short contact heat stimuli selected relative to the subject's pain threshold, because we expected individual differences in the temperature at which the transition from pure heat to heat pain perception occurs.

\section{METHOD}

\section{Subjects}

Twenty-five healthy individuals with a mean age of 28.8 years ( $S D=3.9$ ) were investigated. Eleven were female, and 14 were male. The protocol was approved by an ethics commission.

\section{Apparatus and Procedure}

The stimulator was a temperature-controlled contact thermode with a stimulation surface of $1.6 \times 3.6 \mathrm{~cm}$, mounted on an articulated arm. Contact pressure could be adjusted and was held at $0.4 \mathrm{~N} / \mathrm{cm}^{2}$. The apparatus also included a thermode controller with a microcomputer for managing thermal stimulation and an IBM personal computer for controlling the procedures and giving a basic evaluation. Integrated into the response panel were visual signaling devices. Acoustic cues were delivered by the sound generator of the personal computer. The apparatus (PATH Tester MPI 100; for details, see Galfe, Lautenbacher, Hölzl, \& Strian, 1990) had been developed earlier in the Department of Neurology of the Max Planck Institute of Psychiatry in cooperation with Phywe Systeme GmbH (Göttingen). The subjects sat upright at a table. The thermode was attached to the thenar of the left hand through a slot in the table. The subjects either used the response panel or gave their ratings with their right hands.

Each session started with the determination of the pain threshold: Beginning at $36^{\circ} \mathrm{C}$, eight heat stimuli were applied with a rate of temperature change of $1.6^{\circ} \mathrm{C} / \mathrm{sec}$. The subjects were instructed to press a button as soon as they felt pain. Each time they pressed the button, the temperature returned to the base value at the same rate. The interstimulus intervals lasted at least $10 \mathrm{sec}$. The start of each trial was announced visually and acoustically, but the stimulus was presented with a pseudorandomized delay of between 1 and $3 \mathrm{sec}$. The pain threshold was calculated as the mean of the peak temperatures of the last five stimuli.
In the second part of the experiment, 30 heat stimuli (base temperature, $36^{\circ} \mathrm{C}$; rate of temperature change, $1.6^{\circ} \mathrm{C} / \mathrm{sec}$; sawtooth form) were applied in three blocks of 10 each. The first stimulus of each block had the intensity of the pain threshold and was the standard for the intensity rating. These replications of the standard were used to control for shifts in the adaptation level. The remaining 9 stimuli varied in intensity around the pain threshold in steps of $0.4^{\circ} \mathrm{C}$, the maximum variation being $\pm 1.6^{\circ} \mathrm{C}$. Consequently, each intensity was applied three times in each session. In each block, the order of the 9 stimuli was randomized. Each stimulus trial consisted of a stimulation interval lasting at least $10 \mathrm{sec}$ and until the base temperature was reestablished, as well as a rating interval of $10 \mathrm{sec}$, which also constituted the interstimulus interval. Both intervals were signaled with acoustic and visual cues. The subjects rated the perceived intensity of each stimulus on a visual analogue scale (horizontal, $10 \mathrm{~cm}$ in length) with the left end marked as $0 \%$, the right end as $100 \%$, and the midpoint as $50 \%$. No other points on the scale were marked. The subjects were instructed to rate the standard stimuli (first stimulus in each block) as $50 \%$ and the other stimuli relative to the modulus. The assumption was that this scale had the properties of an interval scale, which measures the subjective deviation of the test sensation from the modulus sensation.

\section{Evaluation}

Because the individual ratings spanned different portions of the full visual analogue scale, we rescaled the ratings $(r)$ with the linear transformation

$$
r^{\prime}=\left(r-r_{\min }\right)\left(100 / r_{\max }-r_{\min }\right),
$$

so that in each case the lowest rating was $0 \%$ and the highest rating was $100 \%$. (Because we assumed only interval properties but no ratio properties for our scale, such a rescaling was possible.) Then, for each of the nine stimulus intensities, we averaged the three ratings $\left(r^{\prime}\right)$ to obtain a mean rating $(R)$ for further evaluation.

We used three types of psychophysical model to describe the relation between the stimulus intensity $(S)$ and the subject's rating $(R)$. The first is a simple model consisting of one linear regression line with two free parameters $(a, b)$ :

$$
R=a S+b .
$$

The second is a power function:

$$
R=a\left(S-S_{0}\right)^{k},
$$

where $S_{0}$ is the estimated "threshold" (correction factor), which has no psychophysical meaning other than its being the estimated stimulus intensity of a $0 \%$ rating, and $k$ is the exponent of the power function. This model has the three free parameters $a, S_{0}$, and $k$. The third model, which we developed to describe the transition from pure heat to heat pain perception, consists of two regression lines, one for the stimulus intensities below and one for those above the measured pain threshold $(C)$ :

$$
R=\begin{array}{ll}
a_{1} S+b_{1} & \text { if } S<C \\
a_{2} S+b_{2} & \text { if } S \geq C
\end{array}
$$

with the condition that

$$
a_{1} C+b_{1}=a_{2} C+b_{2},
$$

where $a_{1}$ and $a_{2}$ are the slopes of the regression lines below and above the measured pain threshold $(C)$, respectively. Because of the condition, the lines intersect at $C$, and TLR has three free parameters just as PF does. 
The psychophysical functions were fitted to the individual and pooled data by the method of least squares. Goodness of fit was evaluated with the use of the coefficient of determination $\left(r^{2}\right)$.

\section{RESULTS}

\section{Psychophysical Parameters Derived From the Individual Data}

Figure 1 shows the individual visual analogue scale ratings as well as the group median and first and third quartiles for each temperature applied. It is clear that most subjects had difficulty differentiating between the two lowest temperatures $\left(-1.6^{\circ} \mathrm{C}\right.$ and $-1.2^{\circ} \mathrm{C}$ below pain threshold); above these temperatures, however, an increase in temperature seems to be monotonically related to an increase in the intensity rating. Correspondingly, except for the two lower stimuli, the ratings of consecutive stimulus intensities were always significantly different (the two lowest, $p=.696$; all others, $p<.01$; Wilcoxon signed-rank test). Only a few subjects had ratings that did not show a strong relation to the temperatures applied.

The slopes of the models with one linear regression line (OLR) and two linear regression lines (TLR) (these slopes give the average change in the rating for a $1^{\circ} \mathrm{C}$ temperature change) and the exponents in the power function (PF) derived from the individual data are given in Table 1. The

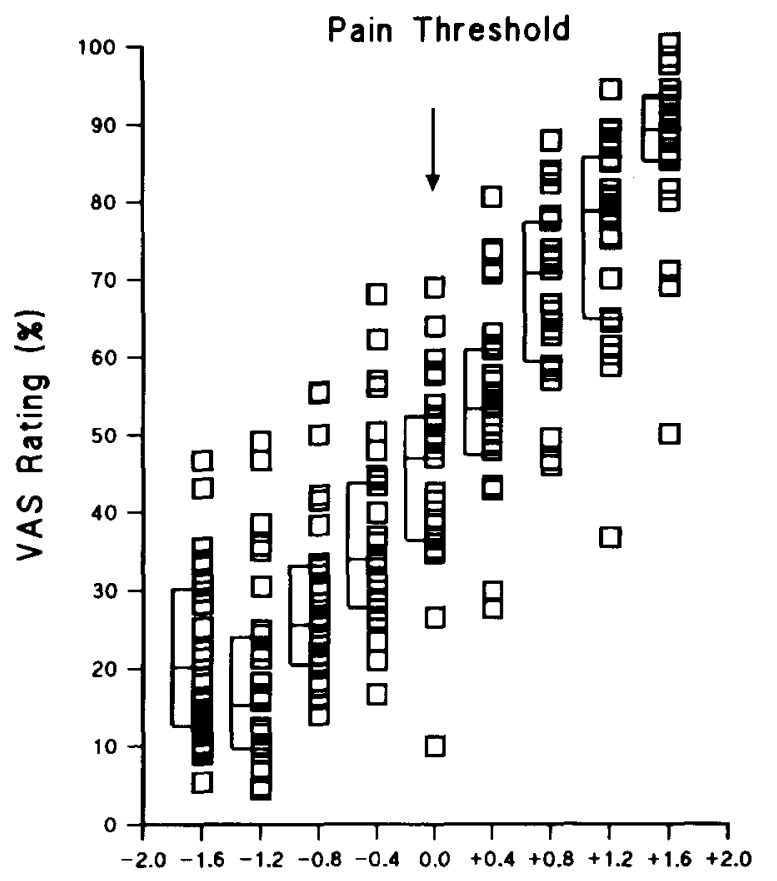

Temperature Relative to Threshold $\left({ }^{\circ} \mathrm{C}\right)$

Figure 1. Relation between temperature (relative to the pain threshold) and rating of intensity on a visual analogue scale (VAS); each square represents the standardized and averaged (see the Method section) rating of one temperature by 1 subject; the two overlapping square brackets indicate quartiles 1 and 3 and the median for that temperature.
Table 1

Slopes in the Models With One Linear Regression Line (a-OLR), Two Linear Regression Lines $\left(a_{1}-\right.$ TLR $=$ Below Pain Threshold, $a_{2}-$ TLR = Above Pain Threshold), and the Exponent of the Model With a Power Function $(k-P F)$, Computed for the Individual (Median, First, and Third Quartiles) and Pooled Data from 25 Subjects

\begin{tabular}{lllll} 
& $a$-OLR & $a_{1}$-TLR & $a_{2}$-TLR & $k$-PF \\
\hline Individual Data & & & & \\
$\quad$ Quartile 1 & 20.37 & 14.74 & 21.37 & 1.10 \\
Median & 21.96 & 18.52 & 27.40 & 1.85 \\
Quartile 3 & 24.88 & 20.96 & 30.25 & 2.65 \\
Pooled Data & 21.80 & 17.13 & 26.47 & 1.89 \\
\hline
\end{tabular}

Table 2

Coefficients of Determination $\left(r^{2}\right)$ Computed as Measures of Goodness of Fit From the Individual and Pooled Data From 25 Subjects for the Models With One Linear Regression Line (OLR), With Two Linear Regression Lines (TLR), and With a Power Function (PF)

\begin{tabular}{llll}
\hline & OLR & TLR & PF \\
\hline Individual Data & & & \\
Quartile 1 & 0.832 & 0.853 & 0.845 \\
Median & 0.907 & 0.933 & 0.934 \\
Quartile 3 & 0.938 & 0.956 & 0.965 \\
Pooled Data & 0.764 & 0.773 & 0.773 \\
\hline
\end{tabular}

slopes in TLR above the pain threshold were significantly larger than those below $(p<.001$, Wilcoxon signed-rank test). Furthermore, compared with the slopes in OLR, those in TLR were significantly smaller below the pain threshold and significantly larger above the pain threshold (for both differences, $p<.001$, Wilcoxon signedrank test). In PF, the exponent values were larger than unity for most of the subjects, which suggests an expansive stimulus/sensation relationship in the transition area from pure heat to heat pain perception.

Table 2 shows the goodness of fit obtained with the three models for the individual data. As expected, the coefficients of determination were significantly larger in both TLR and PF than in OLR (for both differences, $p<$ .001 , Wilcoxon signed-rank test). TLR and PF did not differ in goodness of fit ( $p=.716$, Wilcoxon signed-rank test).

\section{Psychophysical Parameters Derived From the Pooled Data}

The slopes of OLR and TLR and the exponent of PF corresponded quite well to the median values for the individual data (Table 1). The curves of the best-fitting functions for the pooled data are given in Figure 2. From the figure, it is clear that the curves of TLR and PF are quite similar to each other but not to the curve of OLR.

Due to the additional source of variance-that is, the interindividual differences-goodness of fit was poorer with the pooled data than with the individual data (Table 2). Again, TLR and PF explained equal portions of the variance and were superior to OLR, although the differences were quite small. 
For both the individual and the pooled data, TLR and PF were equally good in explaining the stimulus/sensation relationship. Both models suggest that the part of the psychophysical function above the pain threshold has a larger slope than does the part below it.

\section{Correlations Between the Pychophysical Parameters}

The median of the pain thresholds measured was $45.40^{\circ} \mathrm{C}$, with Quartile $1=43.15^{\circ} \mathrm{C}$ and Quartile $3=$ $47.80^{\circ} \mathrm{C}$.

If it is true that the transition from pure heat to heat pain perception has similar psychophysical characteristics in different individuals but that the temperature at which the transition occurs varies from one individual to another, then the correlations between the pain threshold and the parameters of the psychophysical models should be close to zero. The correlations are given in Table 3 . The findings are consistent with this assumption for TLR and PF, but not for OLR, where the pain threshold had a significant positive correlation with the steepness of the slope. Because TLR and PF are superior to OLR in goodness of fit for both the individual and the pooled data, the assumption does not have to be rejected.

Whereas the significant positive correlations between the slope of OLR and the slopes of TLR above and below the pain threshold are not surprising, the correlations

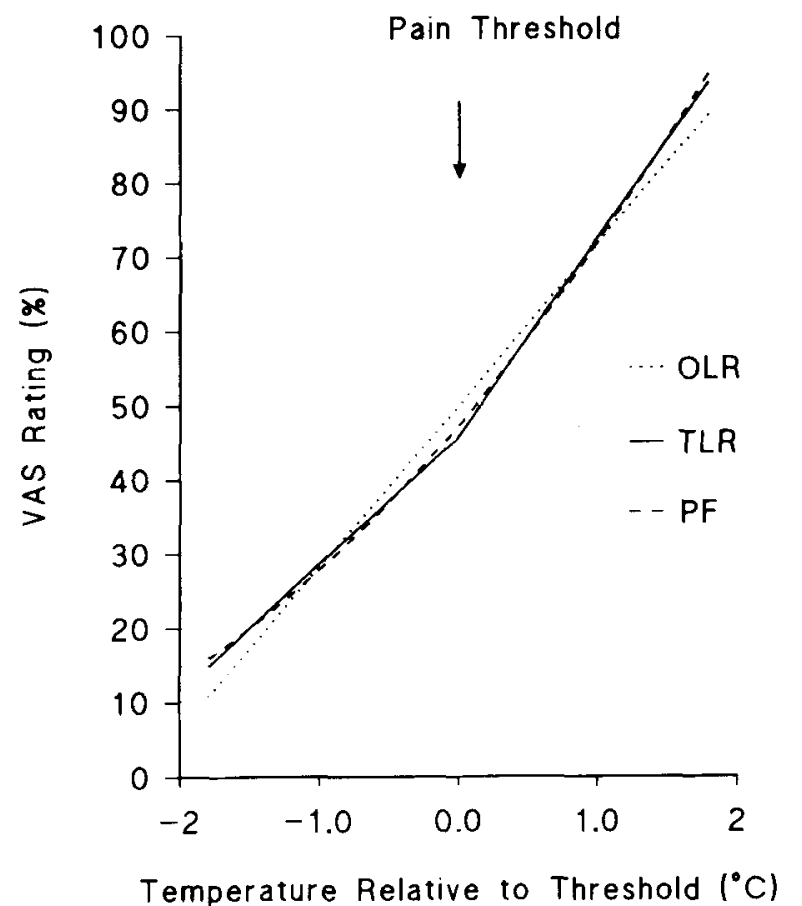

Figure 2. Psychophysical curves for the relation between temperature (relative to the pain threshold) and rating of intensity on a visual analogue scale (VAS) calculated with the three models: with one linear regression line (OLR), with two linear regression lines intersecting at the pain threshold (TLR), and with a power function (PF). Data are pooled from 25 subjects.
Table 3

Spearman Rank Correlations Between the Pain Threshold (PT), the Slopes in the Models With One Linear Regression Line ( $a$-OLR) and Two Linear Regression Lines $\left(a_{1}-\right.$ TLR $=$ Below Pain Threshold, $a_{2}-$ TLR = Above Pain Threshold), and the Exponent of the Model With a Power Function $(k-\mathrm{PF}) ; \boldsymbol{n}=25$ in Each Correlation

\begin{tabular}{lcccc} 
& $a$-OLR & $a_{1}-$ TLR & $a_{2}-$ TLR & $k$-PF \\
\hline PT & $0.414^{*}$ & 0.084 & 0.293 & 0.047 \\
$a$-OLR & & $0.533 \dagger$ & $0.594 \dagger$ & -0.222 \\
$a_{1}$-TLR & & & -0.249 & $-0.879 \ddagger$ \\
$a_{2}$-TLR & & & $0.512 \dagger$ \\
\hline${ }^{*} p \leq .05 . \quad \dagger p \leq .01 . \quad \neq p \leq .001$. & &
\end{tabular}

between the exponent of PF and the slopes of TLR are, especially considering their size (see Table 3). According to these correlations, the exponent of PF had a very close negative relationship to the slope of TLR below the pain threshold. This means that a slow increase in the rating with increases in stimulus intensity in the heat range below the pain threshold was strongly associated with a large exponent in the power function over the whole stimulus range, and vice versa. In other words, with the inclusion of both the heat range and the heat pain range in one psychophysical function, large exponents do not necessarily indicate a strongly expansive relation between stimulus intensity and sensation intensity in the pain range; rather, they seem to result mainly from the slope of the psychophysical curve in the heat range.

\section{DISCUSSION}

The findings in the present study demonstrate that the two models (our model with two linear regression lines intersecting at the pain threshold, and the traditional one with a power function) are equally good in explaining the transition from pure heat to heat pain perception. The reasons why we prefer our model are as follows: First, our model takes into account that there is a change in sensory modality, whereas the exponential model does not. Second, the assumption of linearity, the simplest one in psychophysics, seems to be sufficient to explain both the upper heat range and the lower heat pain range.

As expected, in our model, the slopes were significantly greater in the heat pain range than they were in the pure heat range. This is compatible with a better discrimination ability in the heat pain range, as has already been observed by others (Kenshalo, Anton, \& Dubner, 1989; Robinson, Torebjoerk, \& LaMotte, 1983). The weaker discrimination ability in the heat range might be the reason why our subjects had trouble discriminating the two stimuli with the lowest temperatures, which surely were nonpainful.

To explain our data over the whole stimulus range from $-1.6^{\circ} \mathrm{C}$ below to $+1.6^{\circ} \mathrm{C}$ above the individual pain threshold by a power function, exponents between 1.8 and 1.9 were needed. These exponent values are very similar to those reported by Price and coworkers (Price, 1988; 
Price et al., 1980; Price \& Harkins, 1987; Price et al., 1983), which were around 2.1 and which these authors interpreted as "pain parameters." The fact that the psychophysical functions used by these authors included both the heat range and the heat pain range, as in our study, allows the interpretation that the exponents are not compelling evidence for an expansive stimulus/sensation relationship in the heat pain range. For our results indicate that exponents of this size may also result from a stimulus/sensation relationship with two linear limbs, one in the pure heat range and one in the heat pain range, with a greater slope above the pain threshold than below it.

An inspection of the data presented graphically in several studies on the stimulus/sensation relationship with heat stimuli ranging from nonpainful to painful corroborates this view (Duncan, Bushnell, \& Lavigne, 1989; Duncan, Bushnell, Lavigne, Lavoie, \& Rivest, 1986 [data presented graphically in Price, 1988]; LaMotte \& Campbell, 1978; Price et al., 1980). Straight lines can be fitted to the data quite well until about $45^{\circ} \mathrm{C}-46^{\circ} \mathrm{C}$, and also from these temperatures on, but there is a rather abrupt change to a greater slope in this region. Preselected, absolute temperature scales were used in these studies and not, as in our study, a psychophysically determined temperature scale related to the pain threshold. Therefore, interindividual variations in the location of the pain threshold may have obscured changes in the slopes of the individual data that were actually even more abrupt. The psychophysical data from studies with a more limited stimulus range, either ending (Refinetti, 1989) or starting (Price, Harkins, Rafii, \& Price, 1986; Price, McHaffie, \& Larson, 1989; Price, Von der Gruen, Miller, Rafii, \& Price, 1985) in the pain threshold region, show much smaller changes in slope.

Our finding of a highly significant negative correlation between the slope computed for the pure heat range according to the model with two linear regression lines and the exponent of the power function computed for the whole stimulus range demonstrates that even the lower part of the psychophysical curve had a strong influence on the size of the exponents. This suggests that the "pain" exponents reported by Price and coworkers (Price, 1988; Price et al., 1980; Price \& Harkins, 1987; Price et al., 1983) were also strongly affected by the slope of the psychophysical function in the heat range. Therefore we think that if true pain parameters are to be derived, the pain threshold must be used as the lower limit of the stimulus range.

As mentioned earlier, the view has been expressed (Price, 1988) that different types of heat pain, such as contact and radiation heat pain, have different psychophysical characteristics and in particular different exponents in a power function. We now postulate that these differences can be explained by the fact that in some studies the transition from pure heat perception to heat pain perception is included in the psychophysical function and in others it is not. A promising approach for investigating psychophysical functions including this transition is the use of two-limbed functions that intersect at the pain threshold. A limitation to linear models is not obligatory. However, a reasonable relation between the number of function parameters and the number of available data points must be preserved.

\section{REFERENCES}

Adair, E. R., Stevens, J. C., \& Marks, L. E. (1968). Thermally induced pain, the Dol scale, and the psychophysical power law. American Journal of Psychology, 81, 147-164.

Algom, D., RaphaEl, N., Cohen-RAZ, L. (1986). Integration of noxious stimulation across separate somatosensory communications systems: A functional theory of pain. Journal of Experimental Psychology: Human Perception \& Performance, 12, 92-102.

Algom, D., Raphaeli, N., Cohen-Raz, L. (1987). Pain combines additively across different sensory systems: $A$ further support for the functional theory of pain. Perceptual \& Motor Skills, 65, 619-625.

BECK, C., \& Rosner, B. S. (1968). Magnitude scales and somatic evoked potentials to percutaneous electrical stimulation. Physiology \& Behavior, 3, 947-953.

Bromm, B., \& TREedE, R.-D. (1980). Withdrawal reflex, skin resistance reaction and pain ratings due to electrical stimuli in man. Pain, 9 , 339-354.

Campbell, I. G., Carstens, E., \& Watkins, L. R. (1991). Comparison of human pain sensation and flexion withdrawal evoked by noxious radiant heat. Pain, 45, 259-268.

Cross, D. V., Tursky, B., \& LODGE, M. (1975). The role of regression and range effects in determination of the power function for electric shock. Perception \& Psychophysics, 18, 9-14.

Duncan, G. H., Bushnell, M. C., Lavigne, G. J. (1989). Comparison of verbal and visual analogue scales for measuring the intensity and unpleasantness of experimental pain. Pain, 37, 295-303.

Duncan, G., Bushnell, C., Lavigne, G., Lavoie, M., Rrvest, P. (1986). Comparison of verbal and visual analogue scales for measuring experimental pain. Journal of Dental Research, 65, 307.

Galfe, G., Lautenbacher, S., Hölzl, R., Strian, F. (1990). Diagnosis of small fibre neuropathy: Computer-assisted methods of combined pain and thermal sensitivity determination. Hospimedica, 8(7), $38-48$.

JONES, B., \& GWYNN, M. (1984). Functional measurement scales of painful electric shocks. Perception \& Psychophysics, 35, 193-200.

Kenshalo, D. R., Jr., Anton, F., \& Dubner, R. (1989). The detection and perceived intensity of noxious thermal stimuli in monkey and in human. Journal of Neurophysiology, 62, 429-436.

LaMotte, R. H., \& Campbell, J. N. (1978). Comparison of responses of warm and nociceptive C-fiber afferents in monkey with human judgments of thermal pain. Journal of Neurophysiology, 41, 509-528.

McCallum, P., \& Goldberg, H. (1975). Magnitude scales for electrocutaneous stimulation. Perception \& Psychophysics, 17, 75-78.

Price, D. D. (1988). Psychological \& neural mechanisms of pain. New York: Raven Press.

Price, D. D., Barrell, J. J., Gracely, R. H. (1980). A psychophysical analysis of experimental factors that selectively influence the affective dimension of pain. Pain, 8, 137-149.

Price, D. D. , Harkins, S. W. (1987). Combined use of experimental pain and visual analogue scales in providing standardized measurement of clinical pain. Clinical Journal of Pain, 3, 1-8.

Price, D. D., Harkins, S. W., Rafi, A., \& Price, C. (1986). A simultaneous comparison of Fenantyl's analgesic effects on experimental and clinical pain. Pain, 24, 197-203.

Price, D. D., McGrath, P. A., Rafi, A., Buckingham, B. (1983). The validation of visual analogue scales as ratio scale measures for chronic and experimental pain. Pain, 17, 45-56.

Price, D. D., McHaffie, J. G., \& Larson, M. A. (1989). Spatial summation of heat-induced pain: Influence of stimulus area and spatial separation of stimuli on perceived pain sensation intensity and unpleasantness. Journal of Neurophysiology, 62, $1270-1279$.

Price, D. D., Von der Gruen, A., Miller, J., Rafi, A., \& Pruce, C. 
(1985). A psychophysical analysis of morphine analgesia. Pain, 22, 261-269.

REFINETT, R. (1989). Magnitude estimation of warmth: Intra- and intersubject variability. Perception \& Psychophysics, 46, 81-84.

Robinson, C. J., Torebjoerk, H. E., LaMotte, R. H. (1983). Psychophysical detection and pain ratings of incremental thermal stimuli: A comparison with nociceptor responses in humans. Brain Research, 274, 87-106.

Rollman, G. B., \& Harris, G. (1987). The detectability, discriminability, and perceived magnitude of painful electrical shock. Perception \& Psychophysics, 42, 257-268.
Stam, H. J., Petrusic, W. M., \& Spanos, N. P. (1981). Magnitude scales for cold pressor pain. Perception \& Psychophysics, 29, 612-617. Stevens, S. S. (1960). The psychophysics of sensory functions. American Scientist, 48, 226-253.

Stevens, S. S., Carton, A. S., \& Shickman, G. M. (1958). A scale of apparent intensity of electric shock. Journal of Experimental Psychology, 56, 328-334.

(Manuscript received June 4, 1991;

revision accepted for publication June 6,1992 .) 